\title{
A Season of American Football Is Not Associated with Changes in Plasma Tau
}

Jonathan M. Oliver

Margaret T. Jones

Anthony J. Anzalone

K. Michele Kirk

David A. Gable

Justin T. Repshas

Torie A. Johnson

Kina Höglund

Kaj Blennow

Henrik Zetterberg

\begin{abstract}
American football athletes are routinely exposed to sub-concussive impacts over the course of the season. This study sought to examine the effect of a season of American football on plasma tau, a potential marker of axonal damage. Nineteen National Collegiate Athletic Association (NCAA) football athletes underwent serial blood sampling over the course of the 2014-2015 season at those times in which the number and magnitude of head impacts likely changed. Non-contact sport controls (NCAA men's swim athletes; $n=19$ ) provided a single plasma sample for comparison. No significant differences were observed between control swim athletes and football athletes following a period of non-contact $(p=0.569)$ or a period of contact $(p=0.076)$. Football athletes categorized as starters $(n=11)$ had higher tau concentrations than non-starters $(n=8)$ following a period of non-contact $(p=0.039)$ and contact $(p=0.036)$, but not higher than swimmers $(p=1.000$ and $p=1.000$, respectively). No difference was noted over the course of the season in football athletes, irrespective of starter status. Despite routine head impacts common to the sport of American football, no changes were observed over the course of the season in football athletes, irrespective of starter status. Further, no difference was observed between football athletes and non-contact control swim athletes following a period of non-contact or contact. These data suggest that plasma tau is not sensitive enough to detect damage associated with repetitive sub-concussive impacts sustained by collegiate-level football athletes.
\end{abstract}

\section{Introduction}

Although a high percentage of sports-related concussions go unreported,1 American football is associated with the highest incidence of head injuries among team sports. 2 Unlike other contact sports, where head impacts occur sporadically, American football athletes are routinely exposed to head trauma of variable magnitude and frequency over the course of a season.3-5 In football, the majority of those impacts occur in the absence of an acute concussive injury, are repetitive in nature, and are thus categorized as sub-concussive head trauma. Nonetheless, even in the absence of an 
acute concussive injury, quantifiable neurological damage can be observed when examined via advanced imaging techniques6-10 and fluid biomarker quantification.11-13 Repetitive subconcussive impacts associated with a lifetime of sports participation may lead to neurological impairments such as chronic traumatic encephalopathy (CTE), which also can now be detected via advanced positron emission tomography imaging such as [F18] FDDNP.9,14,15

Among those fluid biomarkers of head trauma, the neuronal protein tau has been well studied.16 Primarily found in axons, tau functions in microtubule assembly and stability. In neurodegenerative disorders such as Alzheimer's disease and CTE, tau proteins are found in a hyperphosporylated state, which leads to the formation of pre-tangles and eventual neurofibrillary tangles.

Hyperphosphorylation of tau results in the failure of tau to bind to tubulin monomers, microtubule disassembly, and impaired axonal transport. Quantification of tau in both cerebrospinal fluid (CSF) and peripheral blood likely indicates a dissociation of the protein from microtubules and is conjectured to be a surrogate marker of head injury severity.16 In fact, CSF tau correlates with 1-year clinical outcomes following severe traumatic brain injury (TBI)17 and elevations in CSF tau are observed following a boxing match in both amateur and Olympic boxers.18,19 The risk and difficulty by which CSF samples are obtained must not be overlooked and recent technological advances have made quantification of tau in the peripheral blood possible. To that end, peripheral tau has been reported to be elevated following a bout of Olympic boxing,18,19 following a sports-related concussion,11,20-22 in previously deployed military personnel with self-reported TBI, and in former National Football League (NFL) athletes.23,24

Given that athletes often do not report to appropriate medical personnel1 and the implications of repetitive sub-concussive impacts to long-term brain health14,15 the elucidation of a peripheral biomarker that would assist in detecting damage associated with sub-concussive injury is of interest. Although CSF tau has been shown to increase following contact sport participation in the absence of a sports-related concussion 11 and to remain elevated for extended periods in those reporting having sustained a TBI, 25 no study has examined the plasma tau in the context of repeat sub-concussive impacts. Therefore, purpose of the current study was to examine peripheral tau concentrations over a 27-week period from off-season training through the competitive sport season in American football athletes.

\section{Methods}

This study was conducted according to the Declaration of Helsinki. All procedures involving human subjects were approved by the Institutional Review board of Texas Christian University for use of human subjects in research. Prior to participation, all athletes involved in the study reviewed and signed an informed consent. 


\section{Participants}

Over the course of the 2014-2015 competitive season, nineteen $(n=19)$ National Collegiate Athletic Association (NCAA) Division 1 football athletes underwent plasma sampling. Prior to the start of the study, athletes were deemed healthy by the team physicians. Athletes who sustained an injury, including concussion, or who were unable to participate in regularly scheduled conditioning or competition were excluded. Non-contact sport controls, NCAA men's swim athletes $(n=19)$, were recruited to obtain a single plasma sample for comparison.

\section{Study design}

To examine changes in plasma tau concentration as a measure of sub-concussive impacts over the course of the season, a longitudinal observational between-group design was employed. Football athletes were classified by starter status; those athletes known to go out with the first or second team (i.e., first or second on the depth roster) were identified as starters. Starters participate in more repetitions per game (i.e., 30-40+ per game) based on respective position, increasing the number of impacts sustained over the course of the season. Eleven starters $(n=11)$ and eight non-starters $(n=8)$ were identified. Further, a between-group design was utilized to examine differences in plasma tau between contact sport athletes known to sustain repetitive sub-concussive impacts regularly (football athletes) and non-contact control athletes (swimmers).

For American football athletes, a fasting blood sample was obtained at eight time-points prior to and during the 2014-2015 NCAA football season. The first blood sample (T1), which was utilized as a baseline, occurred before summer conditioning following a 9-week period of non-contact. Summer conditioning included five weekly strength and conditioning sessions and non-supervised seven-onseven practices that involved no contact or equipment and occurred twice weekly. The duration of summer conditioning was approximately 8 weeks and no head contact or full-speed practice occurred throughout that period. The second blood sample (T2) was obtained immediately following summer conditioning and just prior to the commencement of a 3-week pre-season training camp that began with non-contact practice and progressed to full-contact practices and eventually two-aday practices with full gear. The third blood sample (T3) was obtained just following pre-season camp. The time between $\mathrm{T} 2$ and $\mathrm{T} 3$ (pre-season training camp) is associated with the highest concentration of impacts for American football athletes over the course of the season. Five additional samples, T4 through T8, were obtained throughout the competitive season on the Monday morning following a Saturday game ( $\sim 36-48 \mathrm{~h}$ ) with no more than 28 days separating time-points. Throughout the competitive season, athletes participated in non-contact Sunday practice, full-gear practice on Tuesday, and non-contact Wednesday, Thursday, and Friday practice. During the season, tackles were designated as partial contact (i.e., hit and wrap only), which aimed to reduce head trauma associated with full-contact practice, in order to ensure player safety and health prior to games. 


\section{Blood sampling and preparation}

Fasting ( $\geq 8 \mathrm{~h}$ ) blood samples were collected via venipuncture using standard sterile phlebotomy procedures in EDTA tubes. Samples were subsequently centrifuged for $10 \mathrm{~min}$ at $4^{\circ} \mathrm{C}$ (Allegra X-15R; Beckman Coulter, Brea, $\mathrm{CA}$ ) within 30 min of collection. Plasma was removed via sterile pipette and immediately transferred and stored at $-80^{\circ} \mathrm{C}$.

\section{Plasma tau concentration}

Plasma tau concentration was measured using the Human Total Tau kit on the Simoa HD-1 analyzer (Quanterix, Lexington, MA). The analyses were performed by a board-certified laboratory technician blinded to the study design in one round of experiments using one batch of reagents with intra-assay coefficients of variation below $10 \%$. The lower limit of quantification was $0.85 \mathrm{pg} \cdot \mathrm{mL}-1$.

\section{Statistical analysis}

All data were normally distributed. Differences between football athletes and swim athletes were compared by an independent samples t-test. Since swim athletes were only sampled at one timepoint, the mean concentration of tau obtained at that time-point was compared with the football athletes at two distinct time-points, $\mathrm{T} 1$ and $\mathrm{T} 8$, given that one represented a time following a 9-week period of non-contact and the other following a period of chronic head impacts. A univariate analysis of variance (ANOVA) was used to compare differences at the same time-point among three groups (swim athletes, football athlete starters, and football athlete non-starters) with a Bonferroni correction factor.

A repeated measures ANOVA was used to examine changes over time in tau concentrations among all football athletes, while a two-factor (time $\times$ starter status) ANOVA was used to test the hypothesis that athletes categorized as starters would differ in tau concentrations over the course of the season. Bonferroni correction factor was used for all post hoc comparisons.

Area under the curve (AUC) was calculated by trapezoidal method and difference between football athletes categorized as starters and non-starters was determined by an independent samples t-test. All statistical analyses procedures were conducted with Statistical Package for the Social Sciences (SPSS V.24; IBM Corporation, Armonk, NY). 


\section{Results}

Tau concentrations following a period of non-contact in American football athletes

Mean tau concentrations were not different between football athletes and swim athletes following a period associated with non-contact in football ( $\mathrm{T} 1 ; \mathrm{p}=0.569$; Fig. $1 \mathrm{~A})$. When football players were further divided by starter status, a significant difference $(F 2,35=3.604 ; p=0.038)$ was observed among groups. No difference was noted in tau concentrations between swim athletes

$(3.67 \pm 0.93 \mathrm{pg} \cdot \mathrm{mL}-1)$ and football athletes categorized as non-starters $(2.46 \pm 1.17 \mathrm{pg} \cdot \mathrm{mL}-1$; $\mathrm{p}=0.117)$. However, football athletes categorized as starters had higher mean tau concentrations $(4.09 \pm 1.93 \mathrm{pg} \cdot \mathrm{mL}-1)$, compared with non-starters (mean difference; $95 \% \mathrm{Cl} ; \mathrm{p}=0.039$ ) but not swim athletes $(p=1.000)$.

FIG. 1.

FIG. 1. Tau concentrations in American football and swim athletes following a period of noncontact. (A) Plasma tau following a period of non-contact in football athletes $(n=19, \boldsymbol{\Delta})$ and noncontact control athletes (swimmers; $n=19, \# \times 25 A 0 ;)$. (B) Plasma tau following a period of noncontact in football athletes starters $(n=11, \diamond)$, non-starters $(n=8,0)$, and non-contact control athletes $(n=19, \boldsymbol{m})$. Horizontal lines represent mean and $95 \%$ confidence interval.

Tau concentrations following a period of contact in football athletes

When post-season mean tau from American football athletes was compared with swim athletes, the difference approached significance ( $p=0.076$; Fig. $2 A)$. However, higher mean tau concentrations were observed in the swim athletes $(3.67 \pm 0.93 \mathrm{pg} \cdot \mathrm{mL}-1)$ but not the football athletes $(3.02 \pm 1.22 \mathrm{pg} \cdot \mathrm{mL}-1)$. Taking into account starter status for football athletes at the same time-point resulted in a significant difference among groups ( $F 2,35=5.442 ; p=0.009)$. No difference was observed between football athletes categorized as starters $(3.55 \pm 1.19 \mathrm{pg} \cdot \mathrm{mL}-1)$ and swim athletes ( $3.67 \pm 0.93 \mathrm{pg} \cdot \mathrm{mL}-1 ; \mathrm{p}=1.000$ ), but both had higher mean concentrations of tau than football athletes categorized as non-starters $(2.31 \pm 0.91 \mathrm{pg} \cdot \mathrm{mL}-1 ; \mathrm{p}=0.036$ and $\mathrm{p}=0.009$, respectively; Fig. 2B).

FIG. 2.

FIG. 2. Tau concentrations in American football and swim athletes following a period of contact. (A) Plasma tau following a period of contact in football athletes $(n=19, \mathbf{\Delta})$ and non-contact control athletes (swimmers; $n=19, \mathbf{a}$ ). (B) Plasma tau following a period of contact in football athletes 
starters $(n=11, \diamond)$, non-starters $(n=8,0)$, and non-contact control athletes $(n=19, \mathbf{\square})$. Horizontal lines represent mean and $95 \%$ confidence interval.

Tau changes in football athletes over the course of the season

Compared with at baseline, mean tau concentrations obtained following a period of non-contact did not change over the course of the season, except for a decrease at the conclusion of fall camp (T3; $p=0.047$; Fig. 3A). Examination of changes based on starter status showed that football athletes categorized as starters had higher mean tau concentrations at baseline (T1), as previously reported, but also at the two samplings corresponding to the end of the season $(p=0.048$ and $p=0.025$; Fig. 3B). However, despite those differences, no significant difference was noted across the entire season when starter status was taken into account. Calculation of tau AUC over the course of the entire season resulted in a significantly higher value for football athletes categorized as starters $(671.03 \pm 172.56 \mathrm{pg} \cdot \mathrm{mL}-1 \cdot \mathrm{d})$, compared with non-starters $(508.36 \pm 160.78 \mathrm{pg} \cdot \mathrm{mL}-1 \cdot \mathrm{d} ; \mathrm{p}=0.052$ ).

FIG. 3.

FIG. 3. Plasma tau over the course of a season in American football athletes. (A) Plasma tau changes over the course of the season in football athletes $(n=19, \mathbf{\Delta})$. (B) Plasma tau changes over the course of the season in football athletes of differing starter status (starters, $\downarrow$; non-starters, O). Plasma tau concentrations over the course of the season with longitudinal changes in (C) football starters ( $n=11, \downarrow)$ and (D) non-starters $(n=8,0)$; (a) represents significant difference from T1; (b) represents significant difference between starters and non-starters.

\section{Discussion}

American football athletes are routinely exposed to head impacts that vary in number and magnitude over the course of the season.3-5 Those impacts result in mechanical forces 26 that occasionally may lead to diffuse axonal injury, 27,28 even in the absence of a concussion diagnosis.12,29 Given that the number of sports-related concussions may be grossly underestimated due to underreporting and the fact that sub-concussive impacts may result in axonal damage, the current study sought to examine the effect of pre-season and sport season football on plasma tau in NCAA Division 1 football athletes. To the authors' knowledge, this is the first study to examine the utility of tau in detecting sub-concussive injury associated with American football athletes. The main findings of the current study were that despite having experienced head impacts over several years, football athletes do not have elevated levels of plasma tau. Further, the repetitive sub-concussive impacts sustained over the course of the season do not result in any quantifiable change in plasma tau. These data suggest that plasma tau may not be sensitive enough to detect the axonal insult resulting from sub-concussive impacts in American football athletes. 
The relationship between repetitive head impacts and late life plasma tau levels has recently been illustrated. In fact, in retired NFL players, a higher repetitive head impact exposure, quantified by the cumulative head impact index, 30 predicted higher later-life levels of plasma total tau. 23 Those findings may indicate that long-term exposure to sub-concussive impacts sustained by $\backslash$ football athletes could result in long-term elevations in tau. However, while plasma tau was correlated with repetitive head impact exposure, there were no group differences in tau concentration between former NFL players and control subjects, and T-tau did not predict clinical function.23 While plasma tau differed at baseline and following a period of contact between football athletes of differing starter status, no difference was observed between control swim athletes and football athletes categorized as starters. Further, although the difference between football athletes, irrespective of starter status, and control swim athletes approached significance following a period of contact, the elevated levels were observed in the control swim athletes. A difference was observed in AUC between starters and non-starters; however, the lack of serial measurements in control swim athletes did not allow a direct comparison of AUC between those athletes and the American football starters.

It has previously been reported that serum neurofilament light (Nf-L) may be the most sensitive marker of neuroaxonal injury in concussion.16,18,19,31 To that end, we previously examined serum $\mathrm{Nf}-\mathrm{L}$ in the same athletes and found differences were likely present between American football athletes categorized as starters and control swim athletes, as well as between football athletes of differing starter status following a period of non-contact.12 Further study is warranted in younger athletes (i.e., high school, collegiate) to determine if a correlation exists between repetitive subconcussive impacts and levels of $\mathrm{Nf}-\mathrm{L}$ and tau.

Elevations in plasma tau have been reported 1-6 days following a bout in Olympic boxers in the absence of a concussion diagnosis.11 However, no difference in plasma tau was observed in ice hockey players in the 1-h period following a friendly game.21,32 Following a concussion, plasma tau peaks over the first $12 \mathrm{~h}, 20,32$ with elevations reported 24 and $72 \mathrm{~h}$ post-injury only in those with prolonged return to play 20 and up to $144 \mathrm{~h}$ in those displaying symptoms of post-concussion syndrome. 32 Therefore, despite the high variability among concussed athletes, plasma tau may be most related to the severity of injury and/or impact that affects subsequent outcome. While both Olympic boxers and football athletes are exposed to routine head impacts, the biomechanical forces acting on the head and brain differ, 33 which may have contributed to lack of change observed over the course of the season in football athletes. Still the lack of difference over the course of the season between football athletes of differing starter status is confounding, given that we have previously reported in the same population that elevations in Nf-L occurred coincident with those times in which the number and magnitude of head impacts likely increased in those football athletes categorized as starters.12

There is an increase in blood-brain barrier permeability associated with physical activity that has been suggested to be intensity dependent.34 Given that all athletes in the current study were participating in physical activity throughout the season, only differing in impacts sustained as a result of sport and starter status, these data support that Nf-L is a more sensitive marker of axonal insult in the absence of a concussion diagnosis. Indeed, serum Nf-L levels correlate with measures of diffuse axonal injury, specifically higher trace and lower fractional anisotropy measured by magnetic 
resonance diffusion tensor imaging, 35 measures which also are related to cumulative head impact exposures in high school football athletes. 29 In the CSF, changes in Nf-L exceed those in tau following a bout of boxing in amateur and Olympic-level athletes.18,19 Further, only Nf-L remains elevated for up to 3 months after a bout and correlated with injury severity.19

This study is not without limitations. The sample size of the current study was small. Further, in the absence of a concussion diagnosis, we must rely upon the impact being of sufficient magnitude to elicit a physiological response. Variability in the magnitude and number of impacts exists with in the same team among differing positions.5 Further, a lack of biomechanical, neuroimaging, and neuropsychological data is a limitation. However, we have previously reported in the same population that a season of American football results in an increase in serum $\mathrm{Nf}-\mathrm{L}$ in athletes categorized as starters 12,13 and that the pattern of increase coincided with those periods in which the number of head impacts likely increased, based upon prior reports.3-5 Elevations in plasma tau have been shown to peak in the 12-h period following injury. Therefore, the time of sampling within the current study (approximately 36 to $48 \mathrm{~h}$ ) may be outside the window to observe significant changes as a result of sub-concussive impacts.

Despite the limitations, the data presented herein are novel in that this is the first study to examine the utility of tau in sub-concussive impacts in a population known to experience routine impacts. Given that others have presented data demonstrating that tau is elevated in the immediate time frame post-concussion, these data suggest that the tau is either not sensitive enough to detect subconcussive impacts or that tau may decrease after an initial increase post-injury, which has been reported.20,32 Further study is warranted, as the ability to quantify sub-concussive impact may enable limits to be set on participation to reduce the likelihood of deleterious effects of repetitive sub-concussive impacts on long-term brain health and further reduce the likelihood of a concussion going unreported.

Acknowledgments

The authors would like to thank Texas Christian University athletes and coaches.

Author Disclosure Statement

No competing financial interests exist. 


\section{References}

1

McCrea M., Hammeke T., Olsen G., Leo P., and Guskiewicz K. (2004). Unreported concussion in high school football players: implications for prevention. Clin. J. Sport Med. 14, 13-17. Crossref, Medline, Google Scholar

Daneshvar D.H., Nowinski C.J., McKee A., and Cantu R.C. (2011). The epidemiology of sport-related concussion. Clin. Sports Med. 30, 1-17. Crossref, Medline, Google Scholar

3

Broglio S.P., Schnebel B., Sosnoff J.J., Shin S., Feng X., He X., and Zimmerman J. (2010). The biomechanical properties of concussions in high school football. Med. Sci. Sports Exerc. 42, 2064. Crossref, Medline, Google Scholar

4

Broglio S.P., Surma T., and Ashton-Miller J.A. (2012). High school and collegiate football athlete concussions: a biomechanical review. Ann. Biomed. Eng. 40, 37-46. Crossref, Medline, Google Scholar

5

Crisco J.J., Fiore R., Beckwith J.G., Chu J.J., Brolinson P.G., Duma S., McAllister T.W., Duhaime A.C., and Greenwald R.M. (2010). Frequency and location of head impact exposures in individual collegiate football players. J. Athl. Train. 45, 549-559. Crossref, Medline, Google Scholar

6

Abbas K., Shenk T.E., Poole V.N., Breedlove E.L., Leverenz L.J., Nauman E.A., Talavage T.M., and Robinson M.E. (2015). Alteration of default mode network in high school football athletes due to repetitive subconcussive mild traumatic brain injury: a resting-state functional magnetic resonance imaging study. Brain Connect. 5, 91-101. Link, Google Scholar

7

Amen D.G., Newberg A., Thatcher R., Jin Y., Wu J., Keator D., and Willeumier K. (2011). Impact of playing American professional football on long-term brain function. J. Neuropsych. Clin. N 23, 98106. Crossref, Medline, Google Scholar8 
Bazarian J.J., Zhu T., Zhong J., Janigro D., Rozen E., Roberts A., Javien H., Merchant-Borna K., Abar B., and Blackman E.G. (2014). Persistent, long-term cerebral white matter changes after sports-related repetitive head impacts. PLoS One 9, e94734. Crossref, Medline, Google Scholar

9

Barrio J.R., Small G.W., Wong K.P., Huang S.C., Liu J., Merrill D.A., Giza C.C., Fitzsimmons R.P., Omalu B., and Bailes J. (2015). In vivo characterization of chronic traumatic encephalopathy using [F-18] FDDNP PET brain imaging. Proc. Natl. Acad. Sci. U. S. A. 112, E2039-E2047. Crossref, Medline, Google Scholar

10

Bazarian J.J., Zhong J., Blyth B., Zhu T., Kavcic V., and Peterson D. (2007). Diffusion tensor imaging detects clinically important axonal damage after mild traumatic brain injury: a pilot study. J. Neurotrauma 24, 1447-1459. Link, Google Scholar

11

Neselius S., Zetterberg H., Blennow K., Randall J., Wilson D., Marcusson J., and Brisby H. (2013).

Olympic boxing is associated with elevated levels of the neuronal protein tau in plasma. Brain Inj. 27, 425-433. Crossref, Medline, Google Scholar

12

Oliver J.M., Jones M.T., Kirk K.M., Gable D.A., Repshas J.T., Johnson T.A., Andreasson U., Norgren N., Blennow K., and Zetterberg H. (2016). Serum neurofilament light in American football athletes over the course of a season. J. Neurotrauma 33, 1784-1789. Link, Google Scholar

13

Oliver J.M., Jones M.T., Kirk K.M., Gable D.A., Repshas J.T., Johnson T.A., Andreasson U., Norgren N., Blennow K., and Zetterberg H. (2016). Effect of docosahexaenoic acid on a biomarker of head trauma in American football. Med. Sci. Sport Exerc. 48, 974-982. Crossref, Medline, Google Scholar

Bailes J.E., Petraglia A.L., Omalu B.I., Nauman E., and Talavage T. (2013). Role of subconcussion in repetitive mild traumatic brain injury: a review. J. Neurosurg. 119, 1235-1245. Crossref, Medline, Google Scholar 
McKee A.C., Cairns N.J., Dickson D.W., Folkerth R.D., Keene C.D., Litvan I., Perl D.P., Stein T.D., Vonsattel J.P., and Stewart W. (2016). The first NINDS/NIBIB consensus meeting to define neuropathological criteria for the diagnosis of chronic traumatic encephalopathy. Acta Neuropathol. 131, 75-86. Crossref, Medline, Google Scholar

Zetterberg H., Smith D.H., and Blennow K. (2013). Biomarkers of mild traumatic brain injury in cerebrospinal fluid and blood. Nat. Rev. Neurol. 9, 201-210. Crossref, Medline, Google Scholar

17

Öst M., Nylén K., Csajbok L., Öhrfelt A.O., Tullberg M., Wikkelsö C., Nellgård P., Rosengren L., Blennow K., and Nellgård B. (2006). Initial CSF total tau correlates with 1-year outcome in patients with traumatic brain injury. Neurology 67, 1600-1604. Crossref, Medline, Google Scholar

18

Neselius S., Brisby H., Theodorsson A., Blennow K., Zetterberg H., and Marcusson J. (2012). CSFbiomarkers in Olympic boxing: diagnosis and effects of repetitive head trauma. PLoS One0 7, e33606. Crossref, Medline, Google Scholar

19

Zetterberg H., Hietala M.A., Jonsson M., Andreasen N., Styrud E., Karlsson I., Edman Å., Popa C., Rasulzada A., and Wahlund L.O. (2006). Neurochemical aftermath of amateur boxing. Arch. Neurol. 63, 1277-1280. Crossref, Medline, Google Scholar

20

Gill J., Merchant-Borna K., Jeromin A., Livingston W., and Bazarian J. (2017). Acute plasma tau relates to prolonged return to play after concussion. Neurology 88, 595-602. Crossref, Medline, Google Scholar

21

Shahim P., Mattsson N., Macy E.M., Crimmins D.L., Ladenson J.H., Zetterberg H., Blennow K., and Tegner Y. (2015). Serum visinin-like protein-1 in concussed professional ice hockey players. Brain Inj. 29, 872-876. Crossref, Medline, Google Scholar 
Shahim P., Tegner Y., Adiutori R., Ladenson J.H., Macy E.H., Crimmins D., Mattsson N., Giovannoni G., Kallberg B., Blennow K., Kuhle J., and Zetterberg H. (2014). Serum neurofilament light protein and visin-like protein-1 in concussed professional ice hockey players. Alzheimers Dement. 4, P655.

Crossref, Google Scholar

23

Alosco M.L., Tripodis Y., Jarnagin J., Baugh C.M., Martin B., Chaisson C.E., Estochen N., Song L., Cantu R.C., and Jeromin A. (2017). Repetitive head impact exposure and later-life plasma total tau in former NFL players. Alzheimers Dement. 7, 33-40. Medline, Google Scholar

24

Stern R.A., Tripodis Y., Baugh C.M., Fritts N.G., Martin B.M., Chaisson C., Cantu R.C., Joyce J.A., Shah S., and Ikezu T. (2016). Preliminary study of plasma exosomal tau as a potential biomarker for chronic traumatic encephalopathy. J. Alzheimers Dis. 51, 1099-1109. Crossref, Medline, Google Scholar

25

Bogoslovsky T., Wilson D., Chen Y., Hanlon D., Gill J., Jeromin A., Song L., Moore C., Gong Y., and Kenney K. (2017). Increases of plasma levels of glial fibrillary acidic protein, tau, and amyloid $\beta$ up to 90 days after traumatic brain injury. J. Neurotrauma 34, 66-73. Link, Google Scholar

\section{6}

Crisco J.J., Wilcox B.J., Beckwith J.G., Chu J.J., Duhaime A.C., Rowson S., Duma S.M., Maerlender A.C., McAllister T.W., and Greenwald R.M. (2011). Head impact exposure in collegiate football players. J Biomech 44, 2673-2678. Crossref, Medline, Google Scholar

27

Adams J.H., Doyle D., Ford I., Gennarelli T., Graham D., and McLellan D. (1989). Diffuse axonal injury in head injury: Definition, diagnosis and grading. Histopathology 15, 49-59. Crossref, Medline, Google Scholar

28

Browne K.D., Chen X.H., Meaney D.F., and Smith D.H. (2011). Mild traumatic brain injury and diffuse axonal injury in swine. J. Neurotrauma 28, 1747-1755. Link, Google Scholar 
Davenport E.M., Whitlow C.T., Urban J.E., Espeland M.A., Jung Y., Rosenbaum D.A., Gioia G.A., Powers A.K., Stitzel J.D., and Maldjian J.A. (2014). Abnormal white matter integrity related to head impact exposure in a season of high school varsity football. J. Neurotrauma 31, 1617-1624. Link, Google Scholar

30

Montenigro P.H., Alosco M.L., Martin B.M., Daneshvar D.H., Mez J., Chaisson C.E., Nowinski C.J., Au R., McKee A.C., and Cantu R.C. (2017). Cumulative head impact exposure predicts later-life depression, apathy, executive dysfunction, and cognitive impairment in former high school and college football players. J. Neurotrauma 34, 328-340. Link, Google Scholar

\section{1}

Neselius S., Brisby H., Marcusson J., Zetterberg H., Blennow K., and Karlsson T. (2014). Neurological assessment and its relationship to CSF biomarkers in amateur boxers. PLoS One 9, e99870. Crossref, Medline, Google Scholar

32

Shahim P., Tegner Y., Wilson D.H., Randall J., Skillbäck T., Pazooki D., Kallberg B., Blennow K., and Zetterberg H. (2014). Blood biomarkers for brain injury in concussed professional ice hockey players. JAMA Neurol. 71, 684-692. Crossref, Medline, Google Scholar

33

Viano D.C., Casson I.R., Pellman E.J., Bir C.A., Zhang L., Sherman D.C., and Boitano M.A. (2005). Concussion in professional football: comparison with boxing head impacts-Part 10. Neurosurgery 57, 1154-1172. Crossref, Medline, Google Scholar

Koh S.X. and Lee J.K. (2014). S100B as a marker for brain damage and blood-brain barrier disruption following exercise. Sports Med. 44, 369-385. Crossref, Medline, Google Scholar

35

Ljungqvist J., Zetterberg H., Mitsis M., Blennow K., and Skoglund T. (2016). Serum neurofilament light protein as a marker of diffuse axonal injury: results from a case series study. J. Neurotrauma 34, 1124-1127. Link, Google Scholar 